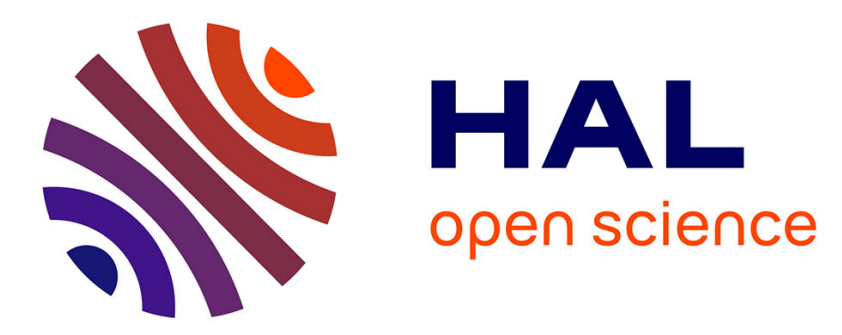

\title{
Higher adaptive optics loop rate enhances axial resolution in nonconfocal ophthalmoscopes
}

Pedro Mecê, Elena Gofas-Salas, Cyril Petit, Frederic Cassaing, José Sahel, Michel Paques, Kate Grieve, Serge Meimon

\section{- To cite this version:}

Pedro Mecê, Elena Gofas-Salas, Cyril Petit, Frederic Cassaing, José Sahel, et al.. Higher adaptive optics loop rate enhances axial resolution in nonconfocal ophthalmoscopes. Optics Letters, 2019, 44 (9), pp.2208. 10.1364/OL.44.002208 . hal-02878515

\section{HAL Id: hal-02878515 https://hal.science/hal-02878515}

Submitted on 23 Jun 2020

HAL is a multi-disciplinary open access archive for the deposit and dissemination of scientific research documents, whether they are published or not. The documents may come from teaching and research institutions in France or abroad, or from public or private research centers.
L'archive ouverte pluridisciplinaire HAL, est destinée au dépôt et à la diffusion de documents scientifiques de niveau recherche, publiés ou non, émanant des établissements d'enseignement et de recherche français ou étrangers, des laboratoires publics ou privés. 


\title{
Higher adaptive optics loop rate enhances axial resolution in nonconfocal ophthalmoscopes
}

\author{
Pedro Mecên ${ }^{1,4}$, Elena Gofas-Salas ${ }^{1,2,4}$, Cyril Petit ${ }^{1,4}$, Frédéric Cassaing ${ }^{1}$, José Sahel 2,3 , \\ Michel Paques ${ }^{2,4}$, Kate Grieve ${ }^{2,4}$, and Serge Meimon ${ }^{1,4}, *$ \\ ${ }^{1}$ ONERA - The French Aerospace Lab, Châtillon, France \\ ${ }^{2}$ Vision Institute, Quinze-Vingts Ophthalmology Hospital, Paris, France \\ ${ }^{3}$ University of Pittsburgh School of Medicine, Pittsburgh, USA \\ ${ }^{4}$ PARIS' Group : Paris Adaptive-optics for Retinal Imaging and Surgery, Paris, France \\ *Corresponding author: pedrofbmece@gmail.com
}

Compiled April 2, 2019

We propose a study to better understand the impact of dynamic ocular aberrations in the axial resolution of nonconfocal adaptive optics $(\mathrm{AO})$ ophthalmoscopes via a simulation of the 3D PSF in the retina for various AOloop rates. We then use Optical Incoherence Tomography, a method enabling the generation of tomographic retinal cross-sections in incoherent imaging systems, to evaluate the benefits of a fast $\mathrm{AO}-\mathrm{loop}$ rate on axial resolution and consequently on AO-corrected retinal image quality. We used the PARIS AO flood-illumination ophthalmoscope for this study, where retinal images from different focal planes at an AO-loop rate of $10 \mathrm{~Hz}$ and $50 \mathrm{~Hz}$ were acquired. (๑) 2019 Optical Society of America

OCIS codes: (330.4460) Ophthalmic optics and devices; (110.1080) Active or adaptive optics; (110.6955) Tomographic imaging.

http://dx.doi.org/10.1364/ao.XX.XXXXXX

\section{INTRODUCTION}

High-resolution imaging of the retina can be achieved using Adaptive Optics (AO) ophthalmoscopes, such as FloodIllumination Ophthalmoscopes (FIO) [1] and Scanning Laser Ophthalmoscopes (SLO) [2], which correct wavefront aberrations introduced by the human eye in real-time. Such highresolution images have an important impact on early-stage retinal disease diagnosis, detection of disease progression and follow-up of therapeutic efficacy at a cellular level [3].

Nonconfocal AO-SLO, i.e. techniques which block directly backscattered light (e.g. dark-field [4], split-detector [5] imaging) or favor multiply scattered components (e.g. offset aperture [6], large pinhole [6], multi-offset [7]), has enabled imaging of retinal structures that were previously poorly visualized, or not visualized at all, such as perfusion in microvasculature [6], ganglion cells [7] and retinal pigment epithelium [4]. The study of these retinal structures has shown their importance to the understanding of diseases such as diabetic retinopathy [8] and age-related macular degeneration (AMD) [9]. Another nonconfo- cal system, the AO-FIO has enabled studying the neurovascular coupling [10] and the evolution of retinal diseases such as AMD and vascular diseases [11] thanks to its inherent advantages over scanning systems of distortionless, widefield and high-speed imaging.

However, compared to the confocal AO-SLO, both nonconfocal AO-SLO and AO-FIO present a significantly lower signal to noise ratio (SNR) and image contrast, which makes them very sensitive to the temporal fluctuation of the ocular aberrations. The dynamic ocular aberrations are mostly due to tear film dynamics and lens fluctuations [12] and are generally greater in patients compared to healthy eyes. To compensate for dynamic aberrations and achieve a better image contrast, Yu et al. implemented a confocal AO-SLO with an increased AO-loop rate (up to $100 \mathrm{~Hz}$ ) [13]. In a dynamic aberration study in 50 eyes, using a high spatiotemporal resolution aberrometer, Jarosz et al. showed that an increase in the AO-loop rate from $10 \mathrm{~Hz}$ to $50 \mathrm{~Hz}$, in nonconfocal ophthalmoscopes, would be beneficial to the image quality [14]. Finally, we recently showed that a factor 4 SNR improvement, at the photoreceptor spatial frequency, is obtained with a $50 \mathrm{~Hz}$ AO-loop rate, compared to $10 \mathrm{~Hz}$, using an $\mathrm{AO}-\mathrm{FIO}$ [15]. Although these studies pointed out the benefits of a highspeed AO-loop rate for the image quality enhancement linked to an improvement in lateral resolution, none of them studied its benefit for the axial resolution. In fact, the axial resolution degradation, due to the presence of aberrations, has been identified in a previous study [16] as a cause of image contrast and SNR loss. However, no study took into account the influence of dynamic aberrations and no appropriate method was used to qualify the axial resolution enhancement for different AO-loop rates. Recently, we introduced a method to generate tomographic retinal cross-sections from en-face images of incoherent based imaging systems, such as nonconfocal ophthalmoscopes, which we named "Optical Incoherence Tomography" (OIT) [17]. Similar to Optical Coherence Tomography (OCT) [18], different retinal layers and structures can be identified, making it a potential tool to visually assess axial resolution of an incoherent imaging system.

In this paper, first we propose a simulation to understand how the AO-corrected axial resolution evolves temporally due 
to dynamic ocular aberrations, and to quantify its degradation for different AO-loop rates. Second, we use the OIT method to evaluate the benefits of a fast AO-loop rate on axial resolution.

\section{METHODS}

To understand how the AO-corrected axial resolution will evolve temporally due to the dynamic ocular aberrations, the PARIS ocular aberrations database, acquired using a high spatiotemporal resolution aberrometer [14], was used. The database consists of 50 series of continuous 3.4 s time sequences, in between blinks, of Zernike coefficients (from a 4 to a45) acquired at $236 \mathrm{~Hz}$ for different healthy eyes. With this data, we can simulate the 3D point spread function (PSF) for each eye and at each time point, for various AO-loop rates (up to $118 \mathrm{~Hz}$ ). This is obtained by computating the 2D PSF associated with the aberrated wavefront, according to standard diffraction theory $[14,19]$, in various defocused planes. Then, we computed the temporal mean value of the axial resolution (defined by the full-width at half maximum) for each eye to draw statistics over the population.

The OIT procedure basically consists of three steps: acquiring en-face images from different focal planes (Z-stack), filtering out the image low-and-mid spatial frequency content using an order 2 Butterworth filter with a normalized cut-off frequency of 0.08 , and obtaining the image sharpness through the computation of the image energy $\left(\sum_{i=1}^{M} \sum_{j=1}^{N}\|\widetilde{I(i, j)}\|^{2}\right.$, where $\widetilde{I}$ is the Fourier Transform of the filtered image of $\mathrm{MxN}$ dimensions). Thanks to the high-pass filter, spatial frequencies coming from out-of-focus layers are filtered out creating a digital optical sectioning effect, since the high-spatial frequency only comes from the in-focus plane [20]. The OIT axial resolution (capacity to separate two retinal layers) is given by the system depth of field, since its metric is defined by the image sharpness [19]. OIT data can be explored in two ways: by the computation of the energy for each image from the Z-stack, generating a retinal axial profile (which we name OIT "A-scan"); and by several energy computations within the field-of-view (FOV) for each image from the Z-stack, generating a tomographic retinal cross-section (which we name OIT "B-scans") (see Visualization 1 for an animation depicting the procedure in detail). From both OIT A-scans and B-scans we can evaluate the axial resolution for different AO-loop rates.

To acquire the Z-stack necessary to generate an OIT image, we used the PARIS AO-FIO [15]. The AO-FIO system comprises two parts that are relevant to this study. The first part is the AO system, which is composed of a superluminescent diode (SLD), with 750nm central wavelength, used as a wavefront sensor (WFS) beacon source; a custom-built Shack-Hartmann WFS, consisting of a $30 \times 30$ microlenslet array, conjugated to the pupil plane, and a $443 \times 443$ pixel complementary metal oxide semiconductor (CMOS) camera (PCO, Edge, Germany) at the focal plane, where aberrations are measured; and a magnetic deformable mirror with 97 actuators (ALPAO, France), which provides wavefront correction thanks to a custom-built real-time calculator closed-loop control. An integrator control strategy, with 0.5 gain and 2-frame delay is used, and AO-loop rate goes up to $70 \mathrm{~Hz}$. The second part is an en-face imaging system composed of a SLD, with a central wavelength of $860 \mathrm{~nm}$; and a scientific CMOS camera (ORCA flash4-V2, Hamamatsu, Japan) which detects back-scattered light from the retina to acquire images of $5.4^{\circ} \times 2.7^{\circ}(2048 \times 1024$ pixel $) \mathrm{FOV}$ at up to $200 \mathrm{~Hz}$. The camera is mounted on a motorized translation stage (Thorlabs, Germany) enabling imaging of various retinal layers. Finally, for optical design reasons unrelated to the current study, the system aperture is limited to a $5 \mathrm{~mm}$ diameter pupil, giving a $38 \mu \mathrm{m}$ depth of field at the diffraction-limit [19] .

Image acquisition was performed on two healthy subjects aged 25 and 23. Research procedures followed the tenets of the Declaration of Helsinki. Informed consent was obtained from subjects after the nature and possible outcomes of the study were explained. Before the acquisition, the subjects' eye was dilated and accommodation paralyzed (one drop of each Tropicamide and Phenylephrine $10 \%$ ), assuring a pupil diameter larger than $5 \mathrm{~mm}$ (full aperture of the system) and a minimal interference of defocus dynamics on the temporal variation of the axial resolution and by consequence on the retinal image quality. Subjects were seated in front of the system and stabilized with a chin and forehead rest and asked to fixate the fixation target. The total power entering the eye from the illumination source and the WFS source were respectively $350 \mu \mathrm{W}$ and $1.8 \mu \mathrm{W}$, which are below the ocular safety limits established by the ISO standards for group 1 devices. Retinal imaging was realized at $7^{\circ}$ and $9^{\circ}$ nasal, where the retinal nerve fiber layer (NFL) is quite dense. For both subjects, a total of 20 image sequences of 100 images were acquired at $200 \mathrm{~Hz}$. Each image sequence was acquired at a specific instrument focal position, generating retinal images from different focal planes. A constant imaging camera translation step of $1.56 \mathrm{~mm}$ (equivalent to a $30 \mu \mathrm{m}$ step in the retinal plane) was adopted, enabling imaging over more than the total retinal thickness. This procedure was done twice, for AO-loop rate set at $10 \mathrm{~Hz}$ and $50 \mathrm{~Hz}$.

Following the acquisition each image sequence was registered using a pairwise maximum likelihood sub-pixel approach [21], where the first image from each sequence was chosen as the reference. After registration, for each sequence, averaged images were computed to improve SNR. After this first processing step, 20 averaged images, of $3^{\circ} \times 2^{\circ} \mathrm{FOV}$, for 20 different instrument focal positions formed a Z-stack of the retina. The Z-stack images were registered two by two using the same approach as before. Finally, each Z-stack image had its low-and-mid spatial frequency filtered out and its energy computed according to the OIT procedure.

\section{RESULTS AND DISCUSSION}

\section{Axial resolution temporal dynamics}

Visualization 2 shows a video of the simulated AO-corrected axial PSF temporal dynamics for various AO-loop rates from one eye of the population. One can notice two effects: 1) an axial displacement, due to the defocus dynamics; 2) an axial elongation due to the high-order aberrations (HOA) dynamics. It is possible to observe that defocus and HOA dynamics have significantly less impact for a higher AO-loop rate. Visualization 2 also helps to understand how dynamic aberrations affect the axial resolution of the imaging system. Images acquired with a long exposure time would lose contrast or SNR, as the axial resolution will be degraded during the image integration. Even if a short exposure time is used, which is the case in this study, final averaged images are produced to increase SNR, leading to elongated axial resolution of the averaged images. This is all the more critical knowing that retinal layer thickness is on the order of tens of micrometers. Although defocus dynamics can be minimized under cycloplegia, as it was done in this study, HOA dynamics can only be controlled with a fast AO-loop rate, as it is shown in Fig. 1 where the average and standard deviation of the axial resolution temporal mean value over the population is drawn. 


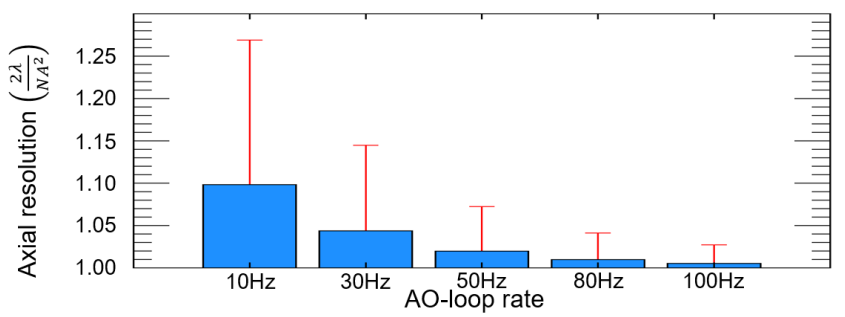

Fig. 1. Average and standard deviation, over the 50-eye population, of the axial resolution temporal mean value for different AO-loop rates. Axial resolution values are given as a multiple of the theoretical diffraction limited resolution.

\section{Axial resolution enhancement visualization in an AO-FIO}

While an axial resolution enhancement can be perceptible in confocal AO-SLO systems, since the axial resolution is proportional to the number of photons collected by the detector, coming from the in-focus retinal layer, the same cannot be said for nonconfocal systems. However, by exploring the digital optical sectioning through the OIT procedure, we are able to evaluate and compare the axial resolution for both AO-loop rates. Figure 2 presents the OIT A-scans obtained for both subjects at $10 \mathrm{~Hz}$ (red line) and $50 \mathrm{~Hz}$ (black line) AO-loop rates.
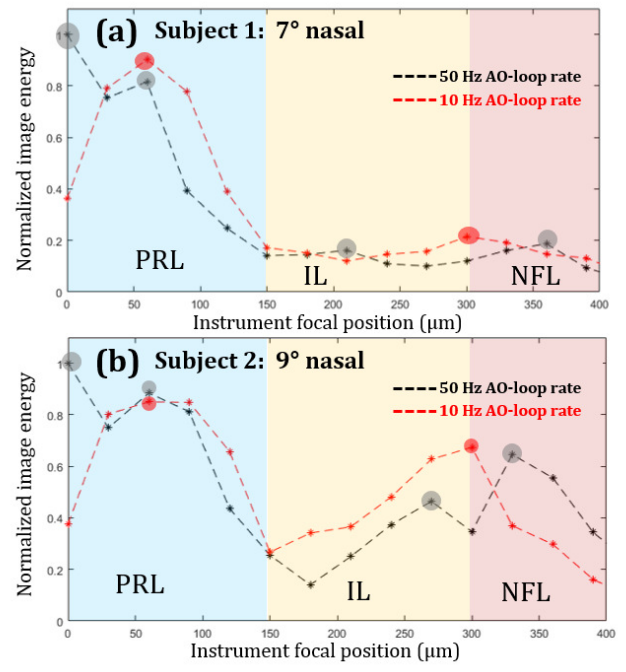

Fig. 2. Image energy (in linear scale) as a function of the instrument focal position using $\mathrm{AO}-\mathrm{loop}$ rates at $50 \mathrm{~Hz}$ (blackdashed line) and $10 \mathrm{~Hz}$ (red-dashed line). (a) and (b) show the results from, respectively, subject 1 (at $7^{\circ}$ nasal), and subject 2 (at $9^{\circ}$ nasal). Red and black circles indicate local maxima. The blue, yellow and red zones correspond to the photoreceptor layer (PRL), intermediate layer (IL), and nerve fiber layer (NFL) respectively.

By visualizing the en-face Z-stack, we categorized the focal positions into three planes, depending on the instrument focal position: the photoreceptor layer (PRL); the intermediate layer (IL), where neither photoreceptor mosaic nor nerve fibers are sharp; and, finally, the NFL. The axial resolution enhancement at $50 \mathrm{~Hz}$ AO-loop rate compared to $10 \mathrm{~Hz}$ is noticed, for both subjects, in two different ways. Firstly, by the image sharpness improvement (higher image energy) for the photoreceptor im- ages (PRL blue region). Secondly, by the increased number of local maxima, since four local maxima can be identified for an AO-loop rate at $50 \mathrm{~Hz}$ (two at the PRL, one at the IL and one at the NFL) against only two at $10 \mathrm{~Hz}$ AO-loop rate (one at the PRL and one at the NFL). The former indicates a lower AO-corrected axial PSF temporal dynamics, leading to an averaged image with enhanced axial resolution. The latter shows that an enhanced axial resolution can increase the number of retinal layers resolved by the OIT.

The same effect can be visually noted in the OIT B-scans. Figures 3 (a) and (b) present the generated OIT B-scans for AOloop rates of $10 \mathrm{~Hz}$ and $50 \mathrm{~Hz}$ respectively from Subject 1 . The number of axial pixels for both images was increased using bicubic interpolation to facilitate visualization. Bright regions represent a higher local sharpness of the corresponding en-face image. Yellow arrows indicate identified layers (considered to be continuous along the image FOV) for both AO-sampling frequencies. Axial resolution enhancement at $50 \mathrm{~Hz}$ AO-loop rate compared to $10 \mathrm{~Hz}$ is noticed by three visual aspects: firstly four layers are identified for $50 \mathrm{~Hz}$ against only two continuous layers for $10 \mathrm{~Hz}$. Secondly, all identified layers are, in general, brighter in the OIT obtained with $50 \mathrm{~Hz}$ AO-loop rate compared to the one at $10 \mathrm{~Hz}$. Finally, a better contrast between bright and dark layers is obtained at $50 \mathrm{~Hz} \mathrm{AO}-$ loop rate.

Figure 3 (c) shows three out of the twenty Z-stack images (those representing the local maxima), where red arrows indicate a transverse vessel. This same vessel is visible in both OIT, but with a higher contrast and axial sectioning at $50 \mathrm{~Hz}$ AO-loop rate OIT. Finally, Fig. 3(d) presents the equivalent OCT, for the same subject and region as OIT B-scans, obtained using Spectralis OCT (Heidelberg, Germany) . Comparing both OIT B-scans with the OCT B-scan, one can notice that while most of retinal layers can be identified in the OIT B-scan acquired with a $50 \mathrm{~Hz}$ AOloop rate, the same cannot be said of the one acquired at $10 \mathrm{~Hz}$ AO-loop rate, since the two PRL bands are confounded, both IL bands are not visible, and NFL does not seem continuous within the FOV.

It should be all the more interesting to analyze the axial resolution enhancement, offered by a fast AO-loop rate, in systems exploring the full eye's pupil aperture (here the system that we used limited this study to a $5 \mathrm{~mm}$ diameter pupil), since dynamic aberrations are higher for a larger aperture, affecting even more the axial resolution. Additionally, our results show that nonconfocal AO-SLO systems, using a dark-field imaging modality for example, can increase the SNR and contrast of the images generated with a faster AO-loop rate, as a better axial resolution would be achieved and more photons from the retinal layer of interest would be collected by the photodetector.

Finally, OIT cannot and does not intend to compete with OCT in terms of image quality - clearly the OCT has higher axial resolution and contrast. However, AO confocal and nonconfocal ophthamoscopes typically have no live axial view for focusing guidance. OIT can provide a true axial/focus representation of the layer structure of the sample being imaged, and can therefore serve as an accurate guidance tool to optimize focus in a particular layer in AO confocal and nonconfocal ophthalmoscopes. This type of guidance tool may be of particular relevance in offset imaging where sets of many low contrast images are acquired and later processed to reveal transparent cells [7], or autofluorescence imaging [22] where SNR on single frames is very low, meaning that during the imaging procedure the operator is essentially "blind" focusing. 

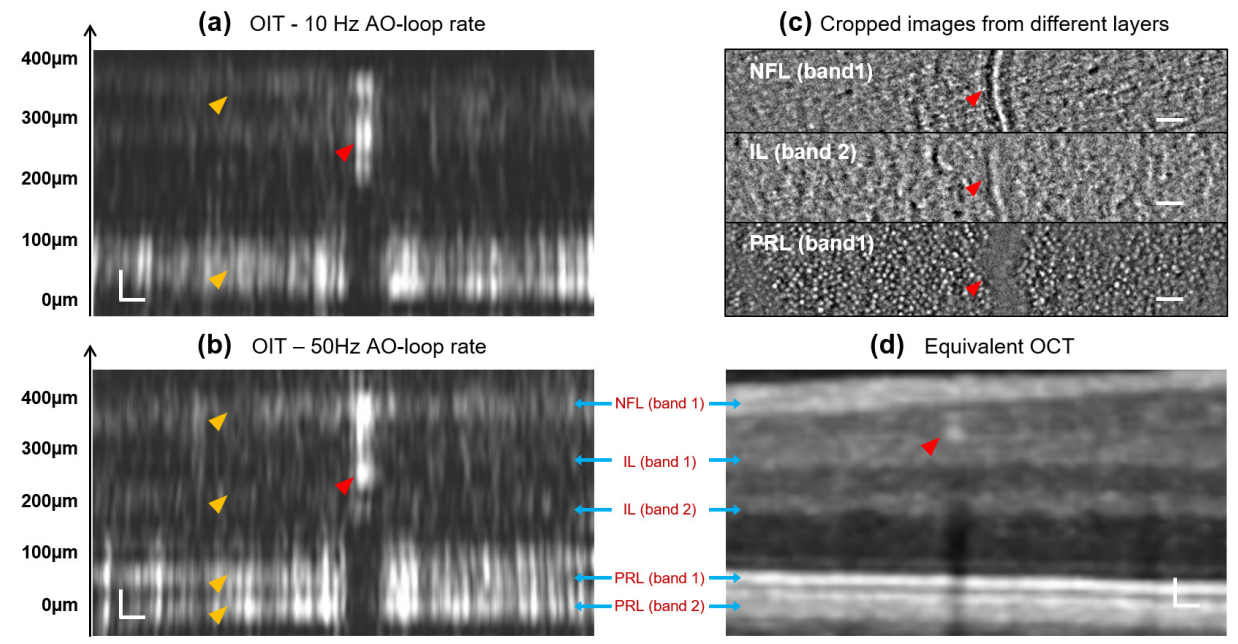

(c) Cropped images from different layers

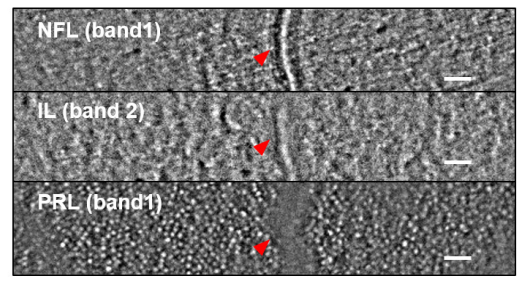

(d) Equivalent OCT

Fig. 3. (a) and (b) generated OIT B-scans (in log scale) for AO-loop rates of $10 \mathrm{~Hz}$ and $50 \mathrm{~Hz}$ respectively for Subject 1 . Yellow arrows indicate identified layers (considered to be continuous along the image FOV) for both AO-sampling frequencies. (c) three out of the twenty Z-stack images (those representing the local maxima/bands), where red arrows indicate a transverse vessel, also axially visible in (a), (b) and (d).(d) the equivalent OCT for the same subject and region as the obtained OIT B-scans. The OCT image was flipped in the horizontal direction to facilitate identification of retinal layers compared to OIT B-scan. Scale bar $50 \mu \mathrm{m}$.

\section{CONCLUSION}

In this study, we demonstrated the potential of the OIT procedure, which digitally performs optical sectioning, to visually assess the axial resolution of an incoherent imaging system with great clarity, making it a highly valuable tool to compare two systems or two settings of the same system in terms of axial resolution or as a focus guidance tool in non-interferometric AO ophthalmoscopes. This procedure was used on the PARIS AO-FIO, comprising a real-time controller up to $70 \mathrm{~Hz}$ loop rate, to evaluate the impact of a high AO-loop rate on axial resolution in a nonconfocal $\mathrm{AO}$ ophthalmoscope. First, we presented a simulation of the temporal behaviour of the 3D PSF in the retina for different AO-loop rates using a high spatiotemporal resolution aberrometer database. This simulation allowed us to understand and quantify the impact of dynamic ocular aberrations on the imaging system axial resolution. Second, we showed that an AO-loop rate of $50 \mathrm{~Hz}$ presents an axial resolution enhancement compared to an AO-loop rate of $10 \mathrm{~Hz}$, thus demonstrating that ocular dynamic aberrations can affect the imaging system axial resolution, leading to retinal images acquired with low contrast and poor SNR. Finally, we conclude that a fast AO-loop rate can increase the OIT image quality, enabling to identify almost all retinal layers using an AO-FIO with a pupil size limited to $5 \mathrm{~mm}$ diameter. This also demonstrates that AO-FIO can indeed achieve decent axial resolution in addition to their inherent advantages over scanning systems of distortionless, wide-field and high-speed imaging.

\section{FUNDING INFORMATION}

Agence Nationale de la Recherche under grants CLOVIS3D (ANR-14-CE17-0011), RHU LIGHT4DEAF (ANR-15-RHUS0001) and ERC Grant Agreement (610110).

\section{REFERENCES}

1. J. Liang, D. R. Williams, and D. T. Miller, JOSA A 14, 2884 (1997).

2. A. Roorda, F. Romero-Borja, W. J. Donnelly III, H. Queener, T. J. Hebert, and M. C. Campbell, Opt. express 10, 405 (2002).
3. P. Godara, A. M. Dubis, A. Roorda, J. L. Duncan, and J. Carroll, Optom. vision science: official publication Am. Acad. Optom. 87, 930 (2010).

4. D. Scoles, Y. N. Sulai, and A. Dubra, Biomed. optics express 4, 1710 (2013).

5. W. Amos, S. Reichelt, D. Cattermole, and J. Laufer, J. microscopy 210, 166 (2003).

6. T. Y. Chui, D. A. VanNasdale, and S. A. Burns, Biomed. optics express 3, 2537 (2012).

7. E. A. Rossi, C. E. Granger, R. Sharma, Q. Yang, K. Saito, C. Schwarz, S. Walters, K. Nozato, J. Zhang, T. Kawakami et al., Proc. Natl. Acad. Sci. 114, 586 (2017).

8. J. Tam, K. P. Dhamdhere, P. Tiruveedhula, S. Manzanera, S. Barez, M. A. Bearse, A. J. Adams, and A. Roorda, Investig. ophthalmology \& visual science 52, 9257 (2011).

9. J. Ambati and B. J. Fowler, Neuron 75, 26 (2012).

10. A. Duan, P. A. Bedggood, A. B. Metha, and B. V. Bui, Sci. reports 7, 2113 (2017).

11. M. Paques, S. Meimon, F. Rossant, D. Rosenbaum, S. Mrejen, F. Sennlaub, and K. Grieve, Prog. retinal eye research (2018).

12. P. Mecê, J. Jarosz, J.-M. Conan, C. Petit, K. Grieve, M. Paques, and S. Meimon, Biomed. optics express 9, 717 (2018).

13. Y. Yu, T. Zhang, A. Meadway, X. Wang, and Y. Zhang, Opt. express 23, 23035 (2015).

14. J. Jarosz, P. Mecê, J.-M. Conan, C. Petit, M. Paques, and S. Meimon, Biomed. optics express 8, 2088 (2017).

15. E. Gofas-Salas, P. Mecê, C. Petit, J. Jarosz, L. M. Mugnier, A. M. Bonnefois, K. Grieve, J. Sahel, M. Paques, and S. Meimon, Appl. Opt. 57, 5635 (2018).

16. F. Romero-Borja, K. Venkateswaran, A. Roorda, and T. Hebert, Appl. optics 44, 4032 (2005).

17. P. Mecê, E. Gofas Salas, C. Petit, K. Grieve, C. Chabrier, M. Paques, and S. Meimon, Proc. SPIE 10858 (2019).

18. D. Huang, E. A. Swanson, C. P. Lin, J. S. Schuman, W. G. Stinson, W. Chang, M. R. Hee, T. Flotte, K. Gregory, C. A. Puliafito et al., Science 254, 1178 (1991).

19. M. Born and E. Wolf, Principles of optics: electromagnetic theory of propagation, interference and diffraction of light (Elsevier, 2013).

20. D. Lim, K. K. Chu, and J. Mertz, Opt. letters 33, 1819 (2008).

21. D. Gratadour, L. Mugnier, and D. Rouan, Astron. \& Astrophys. 443, 357 (2005).

22. K. Grieve, E. Gofas-Salas, R. D. Ferguson, J. A. Sahel, M. Paques, and E. A. Rossi, Biomed. Opt. Express 9, 5946 (2018). 


\section{FULL REFERENCES}

1. J. Liang, D. R. Williams, and D. T. Miller, "Supernormal vision and high-resolution retinal imaging through adaptive optics," JOSA A 14, 2884-2892 (1997).

2. A. Roorda, F. Romero-Borja, W. J. Donnelly III, H. Queener, T. J. Hebert, and M. C. Campbell, "Adaptive optics scanning laser ophthalmoscopy," Opt. express 10, 405-412 (2002).

3. P. Godara, A. M. Dubis, A. Roorda, J. L. Duncan, and J. Carroll, "Adaptive optics retinal imaging: emerging clinical applications," Optom. vision science: official publication Am. Acad. Optom. 87, 930 (2010).

4. D. Scoles, Y. N. Sulai, and A. Dubra, "In vivo dark-field imaging of the retinal pigment epithelium cell mosaic," Biomed. optics express 4 , 1710-1723 (2013).

5. W. Amos, S. Reichelt, D. Cattermole, and J. Laufer, "Re-evaluation of differential phase contrast (dpc) in a scanning laser microscope using a split detector as an alternative to differential interference contrast (dic) optics," J. microscopy 210, 166-175 (2003).

6. T. Y. Chui, D. A. VanNasdale, and S. A. Burns, "The use of forward scatter to improve retinal vascular imaging with an adaptive optics scanning laser ophthalmoscope," Biomed. optics express 3, 25372549 (2012).

7. E. A. Rossi, C. E. Granger, R. Sharma, Q. Yang, K. Saito, C. Schwarz, S. Walters, K. Nozato, J. Zhang, T. Kawakami et al., "Imaging individual neurons in the retinal ganglion cell layer of the living eye," Proc. Natl. Acad. Sci. 114, 586-591 (2017).

8. J. Tam, K. P. Dhamdhere, P. Tiruveedhula, S. Manzanera, S. Barez, M. A. Bearse, A. J. Adams, and A. Roorda, "Disruption of the retinal parafoveal capillary network in type 2 diabetes before the onset of diabetic retinopathy," Investig. ophthalmology \& visual science $\mathbf{5 2}$, 9257-9266 (2011).

9. J. Ambati and B. J. Fowler, "Mechanisms of age-related macular degeneration," Neuron 75, 26-39 (2012).

10. A. Duan, P. A. Bedggood, A. B. Metha, and B. V. Bui, "Reactivity in the human retinal microvasculature measured during acute gas breathing provocations," Sci. reports 7, 2113 (2017).

11. M. Paques, S. Meimon, F. Rossant, D. Rosenbaum, S. Mrejen, F. Sennlaub, and K. Grieve, "Adaptive optics ophthalmoscopy: Application to age-related macular degeneration and vascular diseases," Prog. retinal eye research (2018).

12. P. Mecê, J. Jarosz, J.-M. Conan, C. Petit, K. Grieve, M. Paques, and S. Meimon, "Fixational eye movement: a negligible source of dynamic aberration," Biomed. optics express 9, 717-727 (2018).

13. Y. Yu, T. Zhang, A. Meadway, X. Wang, and Y. Zhang, "High-speed adaptive optics for imaging of the living human eye," Opt. express 23, 23035-23052 (2015).

14. J. Jarosz, P. Mecê, J.-M. Conan, C. Petit, M. Paques, and S. Meimon, "High temporal resolution aberrometry in a 50 -eye population and implications for adaptive optics error budget," Biomed. optics express 8 , 2088-2105 (2017).

15. E. Gofas-Salas, P. Mecê, C. Petit, J. Jarosz, L. M. Mugnier, A. M. Bonnefois, K. Grieve, J. Sahel, M. Paques, and S. Meimon, "High loop rate adaptive optics flood illumination ophthalmoscope with structured illumination capability," Appl. Opt. 57, 5635-5642 (2018).

16. F. Romero-Borja, K. Venkateswaran, A. Roorda, and T. Hebert, "Optical slicing of human retinal tissue in vivo with the adaptive optics scanning laser ophthalmoscope," Appl. optics 44, 4032-4040 (2005).

17. P. Mecê, E. Gofas Salas, C. Petit, K. Grieve, C. Chabrier, M. Paques, and S. Meimon, "Visualizing and enhancing axial resolution in nonconfocal adaptive optics ophthalmoscopy," Proc. SPIE 10858 (2019).

18. D. Huang, E. A. Swanson, C. P. Lin, J. S. Schuman, W. G. Stinson, W. Chang, M. R. Hee, T. Flotte, K. Gregory, C. A. Puliafito et al., "Optical coherence tomography," Science 254, 1178-1181 (1991).

19. M. Born and E. Wolf, Principles of optics: electromagnetic theory of propagation, interference and diffraction of light (Elsevier, 2013).

20. D. Lim, K. K. Chu, and J. Mertz, "Wide-field fluorescence sectioning with hybrid speckle and uniform-illumination microscopy," Opt. letters 33, 1819-1821 (2008).

21. D. Gratadour, L. Mugnier, and D. Rouan, "Sub-pixel image registration with a maximum likelihood estimator-application to the first adaptive optics observations of arp 220 in the I band," Astron. \& Astrophys. 443, 357-365 (2005).

22. K. Grieve, E. Gofas-Salas, R. D. Ferguson, J. A. Sahel, M. Paques, and E. A. Rossi, "In vivo near-infrared autofluorescence imaging of retinal pigment epithelial cells with $757 \mathrm{~nm}$ excitation," Biomed. Opt. Express 9, 5946-5961 (2018). 